\title{
NORMALITY OF SOME $p$-ADIC PRODUCT EXPANSIONS
}

\author{
ARNOLD KNOPFMACHER and JOHN KNOPFMACHER
}

(Received 6 July 1989)

Communicated by J. H. Loxton

\begin{abstract}
We consider two unique products

$$
x=\prod_{n=1}^{\infty}\left(1+a_{n} p^{n}\right), \quad x=\prod_{n=1}^{\infty}\left(1+p^{n}\right)^{b_{n}}
$$

for a given $p$-adic integer $x$ with leading coefficient 1 , where $a_{n}, b_{n} \in\{0,1, \ldots, p-1\}$. It is shown that, for almost all such $x$ relative to Haar measure on the $p$-adic integers, the sequences $\left(a_{n}\right),\left(b_{n}\right)$ are normal to base $p$, and have standard normal distribution functions.
\end{abstract}

1980 Mathematics subject classification (Amer. Math. Soc.) (1985 Revision): 11 K 41, 11 K 55.

\section{Introduction}

Let $\mathbb{Q}_{p}$ denote the field of $p$-adic numbers, that is, the completion of the rational field $\mathbb{Q}$ relative to the usual $p$-adic valuation ||$_{p}$. Consider the circle ("disc", "ball")

$$
\mathfrak{P}_{1}=1+p \mathbb{Z}_{p}=C\left(1, p^{-1}\right)
$$

with centre 1 and radius $p^{-1}$ in $\mathbb{Q}_{p}$, where following Sprindžuk [7, page 67] we define the circle $C(x, r)$ with centre $x$ and radius $r$ to be $\{y \in$ $\left.\mathbb{Q}_{p}:|x-y|_{p} \leq r\right\}$. Then $\mathfrak{P}_{1}$ is the set of all $p$-adic integers with $p$-adic expansions of type $1+\sum_{n=1}^{\infty} c_{n} p^{n}$, where $c_{n} \in \Gamma_{p}:=\{0,1, \ldots, p-1\}$.

Recently the present authors [2,3] established the existence of unique convergent product representations (relative to ||$_{p}$ ) for any $x \in \mathfrak{P}_{1}$, which

(C) 1990 Australian Mathematical Society 0263-6115/90 \$A2.00+0.00 
can be written in the forms

$$
x=\prod_{n=1}^{\infty}\left(1+a_{n} p^{n}\right), \quad \text { and } \quad x=\prod_{n=1}^{\infty}\left(1+p^{n}\right)^{b_{n}},
$$

where $a_{n}, b_{n} \in \Gamma_{p}$. These representations are therefore product analogues of the standard series representations $\sum_{n=0}^{\infty} c_{n} p^{n}, c_{n} \in \Gamma_{p}$, for $p$-adic integers.

Ruban [6] has outlined proofs that the following analogues of properties of "decimal" expansions of real numbers are valid for the standard series representation of $p$-adic integers $x=\sum_{n=0}^{\infty} c_{n}(x) p^{n}$ :

(i) the "digit" functions $c_{n}: \mathbb{Z}_{p} \rightarrow \Gamma_{p}$ are identically-distributed and independent random variables relative to Haar measure $\mu$ on $\mathbb{Z}_{p}$, with $\mu\left\{x: c_{n}(x)=k\right\}=p^{-1}$ for each $n \geq 1$ and each $k \in \Gamma_{p}$;

(ii) relative to Haar measure on $\mathbb{Z}_{p}$, almost all $p$-adic integers $x$ have the same asymptotic frequency

$$
p^{-1}=\lim _{n \rightarrow \infty} \frac{1}{n} \#\left\{r \leq n: c_{r}(x)=k\right\}
$$

for each possible digit value $k \in \Gamma_{p}$.

The latter result of Ruban is of course a $p$-adic analogue of Borel's theorem on "simply" normal real numbers, and it is natural to ask whether the abovestated product representations have similar properties. The main purpose of the present paper is to establish such results, as well as further ones including some involving error estimates and also general normality (to base $p$ ).

\section{Normality of the simple product}

We shall refer to $x=\prod_{n=1}^{\infty}\left(1+a_{n} p^{n}\right), a_{n}=a_{n}(x) \in \Gamma_{p}$, as the simple product for $x \in \mathfrak{P}_{1}$. Properties like its existence with unique $p$-adic digits $a_{n}(x)$ for given $x \in \mathfrak{P}_{1}$, and the convergence of arbitrary products of this type to elements of $\mathfrak{P}_{1}$, were treated in [2]. In particular

$$
1+b_{1} p^{r_{1}}+b_{2} p^{r_{2}}+\cdots=\left(1+b_{1} p^{r_{1}}\right)\left(1+b_{2} p^{r_{2}}\right) \cdots,
$$

if $1 \leq r_{1}<r_{2}$ and $b_{1}, b_{2} \in\{1, \ldots, p-1\}$, but later factors of the product need not always conform to this initial pattern.

The preceding conclusions also imply that there exist well-defined operators $a: \mathfrak{P}_{1} \rightarrow \Gamma_{p}$ and $T: \mathfrak{P}_{1} \rightarrow \mathfrak{P}_{1}$, with $a(x)=a_{1}(x)$ and $T(x)=$ $\prod_{n=1}^{\infty}\left(1+a_{n+1}(x) p^{n}\right)$ for $x \in \mathfrak{P}_{1}$. Then $a_{2}(x)=a(T(x))$ and more generally $a_{r}(x)=a\left(T^{r-1}(x)\right)$. By first proving that the operator $T$ is ergodic relative to Haar measure on $\mathbb{Z}_{p}$, it is possible to deduce that almost every 
$x \in \mathfrak{P}_{1}$ has a "simply normal" product expansion as above, in the sense that the asymptotic frequency

$$
\lim _{n \rightarrow \infty} \frac{1}{n} \#\left\{r \leq n: a_{r}(x)=k\right\}=p^{-1}
$$

for each digit $k \in \Gamma_{p}$. Instead, however, we shall use probability theory and only minor properties of $T$, in order to derive error terms for such limits, and also other results including the general normality to base $p$ of the sequence $\left(a_{r}(x)\right)$ for almost every $x \in \mathfrak{P}_{1}$. Here, as in Postnikov [5, page 62], we define a sequence of integers $a_{r} \in \Gamma_{q}:=\{0,1, \ldots, q-1\}$ to be normal to base $q>1$ if and only if

$$
\lim _{n \rightarrow \infty} \frac{1}{n} \#(\Delta ; n)=q^{-t}
$$

for every $t \geq 1$ and every block $\Delta=\left(b_{1}, \ldots, b_{t}\right)$ of "digits" $b_{i} \in\{0,1, \ldots$, $q-1\}$ where \#( $\Delta ; n)$ is the number of appearances of $\Delta$ as a block $\left(a_{r}, \ldots\right.$, $\left.a_{r+t-1}\right)$ in the sequence $\left(a_{1}, a_{2}, \ldots, a_{n}\right)$.

Now consider the Haar measure $\mu$ on $\mathbb{Z}_{p}$, normalized so that $\mu\left(\mathfrak{P}_{1}\right)=$ $\mu\left(p \mathbb{Z}_{p}\right)=1$. A convenient description of $\mu$ is given in Sprindžuk [7, pages 67-70]. In particular $\mu(C)=p^{-n}$ for any circle $C(x, r)$ of radius $r=$ $p^{-n-1}$ as before, and $\mu\left(\mathfrak{P}_{1}\right)=1$ since $\mathfrak{P}_{1}=C\left(1, p^{-1}\right)$.

Next put $I_{0}=\mathfrak{P}_{1}$, and, for any given digits $k_{1}, \ldots, k_{n} \in \Gamma_{p}$, let

$$
I_{n}=I_{n}\left(k_{1}, \ldots, k_{n}\right)=\left\{x \in \mathfrak{P}_{1}: a_{1}(x)=k_{1}, \ldots, a_{n}(x)=k_{n}\right\} .
$$

We shall call $I_{n}$ a (simple) basic cylinder of rank $n$. Any $x \in I_{n}$ has the form $x=\omega_{n} \prod_{r=n+1}^{\infty}\left(1+a_{r} p^{r}\right)$, where $\omega_{n}=\prod_{r=1}^{n}\left(1+k_{r} p^{r}\right)$, and we obtain a map $\psi_{n}=\psi_{n}\left(k_{1}, \ldots, k_{n}\right): \mathfrak{P}_{1} \rightarrow I_{n}$ if we define

$$
\psi_{n}(y)=\omega_{n} \prod_{r=n+1}^{\infty}\left(1+a_{r-n}(y) p^{r}\right) .
$$

Then $\psi_{n}\left(T^{n}(x)\right)=x$ for $x \in I_{n}$, and $T^{n}\left(\psi_{n}(y)\right)=y$ for $y \in \mathfrak{P}_{1}$, so that $\psi_{n}$ is inverse to $T^{n} \mid I_{N}$. Further, since (1.1) implies that the circle

$$
C\left(1, p^{-n-1}\right)=\left\{y \in \mathbb{Q}_{p}: y=\prod_{r=n+1}^{\infty}\left(1+a_{r} p^{r}\right) \text { for digits } a_{r} \in \Gamma_{p}\right\},
$$

it follows that

$$
I_{n}=\operatorname{Im}\left(\psi_{n}\right)=\omega_{n} C\left(1, p^{-n-1}\right)=C\left(\omega_{n}, p^{-n-1}\right) .
$$

Thus $\mu\left(I_{n}\right)=p^{-n}$. 
For $n \geq 2$ we then obtain

$$
\begin{aligned}
\mu\{x & \left.\in \mathfrak{P}_{1}: a_{2}(x)=k_{2}, \ldots, a_{n}(x)=k_{n}\right\}=\mu\left(\bigcup_{k_{1} \in \Gamma_{p}} I_{n}\left(k_{1}, k_{2}, \ldots, k_{n}\right)\right) \\
& =\sum_{k_{1} \in \Gamma_{p}} \mu\left(I_{n}\left(k_{1}, \ldots, k_{n}\right)\right)=p^{1-n} .
\end{aligned}
$$

Similarly, for any indices $r_{1}<\cdots<r_{n}$, we deduce that

$$
\mu\left\{x \in \mathfrak{P}_{1}: a_{r_{1}}(x)=k_{1}, \ldots, a_{r_{n}}(x)=k_{n}\right\}=p^{-n} .
$$

This shows that the digit functions $a_{r}: \mathfrak{P}_{1} \rightarrow \Gamma_{p}$ are identically-distributed and independent random variables relative to $\mu$, with $\mu\left\{x \in \mathfrak{P}_{1}: a_{r}(x)=\right.$ $k\}=p^{-1}$ for each $r \geq 1$ and each $k \in \Gamma_{p}$. In fact this deduction extends, for any $t \geq 1$, to the functions $a_{t, r}: \mathfrak{P}_{1} \rightarrow \Gamma_{q}$ with $q=p^{t}$, defined as follows

$$
a_{t, 1}(x)=\sum_{M=1}^{t} a_{M}(x) p^{M-1}, \quad a_{t, 2}(x)=\sum_{M=t+1}^{2 t} a_{M}(x) p^{M-t-1},
$$

and so on. Then each $a_{t, r}(x) \in \Gamma_{q}=\{0,1, \ldots, q-1\}$, and (1.3) implies that, for any $q$-adic digits $h_{1}, \ldots, h_{n} \in \Gamma_{q}$, and any indices $r_{1}<\cdots<r_{n}$,

$$
\mu\left\{x \in \mathfrak{P}_{1}: a_{t, r_{1}}(x)=h_{1}, \ldots, a_{t, r_{n}}(x)=h_{n}\right\}=p^{-t n}=q^{-n} \text {. }
$$

Thus the $q$-adic digit functions $a_{t, r}: \mathfrak{P}_{1} \rightarrow \Gamma_{q}$ are identically-distributed and independent random variables relative to $\mu$, with $\mu\left\{x \in \mathfrak{P}_{1}: a_{t, r}(x)=\right.$ $h\}=q^{-1}$ for each $r \geq 1$ and each $h \in \Gamma_{q}$.

Given any $h \in \Gamma_{q}$, now define new random variables $\varepsilon_{t, r, h}$ on $\mathfrak{P}_{1}$ by letting $\varepsilon_{t, r, h}(x)=1$ if $a_{t, r}(x)=h$, and $\varepsilon_{t, r, h}(x)=0$ otherwise.

Then, relative to $\mu, \varepsilon_{t, r, h}$ has the same expected value $q^{-1}$, and variance $(q-1) q^{-2}$, for each $r \geq 1$ and $h \in \Gamma_{q}$. Therefore, by standard theorems on sums of independent and identically-distributed random variables (compare [1, Theorems 3.16-3.18]) we obtain

(1.4) Theorem. For any $h \in \Gamma_{q}$, and almost every $x \in \mathfrak{P}_{1}$,

$$
\frac{1}{n} \#\left\{r \leq n: a_{t, r}(x)=h\right\}=q^{-1}+O\left(\sqrt{\frac{\log \log (n / q)}{n q}}\right) \text {. }
$$

Furthermore, for any real $z$,

$$
\mu\left\{x \in \mathfrak{P}_{1}: \#\left\{r \leq n: a_{t, r}(x)=h\right\}-\frac{n}{q}<\frac{z}{q} \sqrt{n(q-1)}\right\} \rightarrow \Phi(z)
$$


as $n \rightarrow \infty$, where $\Phi(z)=(1 / \sqrt{2 \pi}) \int_{-\infty}^{z} e^{-u^{2} / 2} d u$ is the standard normal distribution function.

It follows from the first assertion of Theorem 1.4 that

$$
\lim _{n \rightarrow \infty} \frac{1}{n} \#\left\{r \leq n: a_{t, r}(x)=h\right\}=q^{-1} \text { a.e. }
$$

for each $h \in \Gamma_{q}$. This amounts to the assertion that, for any $t \geq 1$ and almost every $x \in \mathfrak{P}_{1}$, the sequence $\left(a_{t, r}(x)\right)$ is "simply" normal to base $q=p^{t}$. By one half of a standard theorem (compare [4, Theorem 8.3]) which is usually stated for decimal-type expansions of real numbers, but amounts to a purely combinatorial assertion (with a combinatorial proof) about sequences of digits, this conclusion about all the sequences $\left(a_{t, r}(x)\right)$ now yields

(1.5) TheOREM. For almost every $x \in \mathfrak{P}_{1}$, the sequence $\left(a_{r}(x)\right)$ of simpleproduct digits of $x$ is normal to base $p$, in the sense of (1.2).

\section{The binomial product}

Now consider the binomial product $x=\prod_{n=1}^{\infty}\left(1+p^{n}\right)^{b_{n}}$ for $x \in \mathfrak{P}_{1}$, with unique $p$-adic digits $b_{n}=b_{n}(x) \in \Gamma_{p}$, having basic properties [3] quite similar to those of the earlier (simple) product. In particular

$$
1+b p^{r}+\text { higher terms }=\left(1+p^{r}\right)^{b} \prod_{n>r}\left(1+p^{n}\right)^{b_{n}}
$$

if $1 \leq r, b \in\{1, \ldots, p-1\}$, but later factors of the binomial product need not always conform to this initial pattern.

In view of the direct parallel between the basic properties of the simple and binomial products, it is now possible to essentially translate the earlier discussion in order to derive directly parallel conclusions. In particular, Theorems 1.4-1.5 carry over to the sequence $\left(b_{r}(x)\right)$ of binomial-product digits of $x \in \mathfrak{P}_{1}$, showing amongst other facts that this sequence is also normal to base $p$, for almost every $x$. Further details are omitted here.

\section{References}

[1] J. Galambos, Representations of real numbers by infinite series, (Springer, 1976).

[2] A. Knopfmacher and J. Knopfmacher, 'A product expansion in $p$-adic and other nonarchimedean fields', Proc. Amer. Math. Soc. 104 (1988), 1031-1035. 
[3] A. Knopfmacher and J. Knopfmacher, 'A binomial product representation for $p$-adic numbers', Arch. Math. 52 (1989), 333-336.

[4] L. Kuipers and H. Niederreiter, Uniform distribution of sequences, (Wiley, 1974).

[5] A. G. Postnikov, Ergodic problems in the theory of congruences and of diophantine approximations, (Amer. Math. Soc., 1967).

[6] A. A. Ruban,'Some metric properties of the p-adic numbers', Siberian Math. J. 11 (1970), 176-180.

[7] V. G. Sprindžuk, Mahler's problem in metric number theory, (Amer. Math. Soc., 1969).

Department of Computational and

Applied Mathematics

University of the Witwatersrand

Johannesburg, Wits 2050

South Africa
Department of Mathematics University of the Witwatersrand Johannesburg, Wits 2050 South Africa 